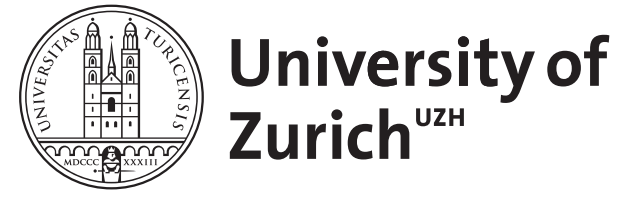

\title{
Mate-sampling costs and sexy sons
}

\author{
Kokko, H ; Booksmythe, I ; Jennions, M D
}

\begin{abstract}
Costly female mating preferences for purely Fisherian male traits (i.e. sexual ornaments that are genetically uncorrelated with inherent viability) are not expected to persist at equilibrium. The indirect benefit of producing 'sexy sons' (Fisher process) disappears: in some models, the male trait becomes fixed; in others, a range of male trait values persist, but a larger trait confers no net fitness advantage because it lowers survival. Insufficient indirect selection to counter the direct cost of producing fewer offspring means that preferences are lost. The only well-cited exception assumes biased mutation on male traits. The above findings generally assume constant direct selection against female preferences (i.e. fixed costs). We show that if mate-sampling costs are instead derived based on an explicit account of how females acquire mates, an initially costly mating preference can coevolve with a male trait so that both persist in the presence or absence of biased mutation. Our models predict that empirically detecting selection at equilibrium will be difficult, even if selection was responsible for the location of the current equilibrium. In general, it appears useful to integrate mate sampling theory with models of genetic consequences of mating preferences: being explicit about the process by which individuals select mates can alter equilibria.
\end{abstract}

DOI: https://doi.org/10.1111/jeb.12532

Posted at the Zurich Open Repository and Archive, University of Zurich ZORA URL: https://doi.org/10.5167/uzh-104936

Journal Article

Accepted Version

Originally published at:

Kokko, H; Booksmythe, I; Jennions, M D (2015). Mate-sampling costs and sexy sons. Journal of Evolutionary Biology, 28(1):259-266.

DOI: https://doi.org/10.1111/jeb.12532 


\section{Mate-sampling costs and sexy sons}

2 Hanna Kokko* ${ }^{1,2,3}$, Isobel Booksmythe ${ }^{4}$, Michael D. Jennions ${ }^{1}$

3

$4{ }^{1}$ Centre of Excellence in Biological Interactions, Evolution, Ecology and Genetics, Research

5 School of Biology, Australian National University, Canberra, Australia

$6 \quad{ }^{2}$ Wissenschaftskolleg zu Berlin, Wallotstrasse 19, 14193 Berlin, Germany

$7{ }^{3}$ Institute of Evolutionary Biology and Environmental Sciences, University of Zurich,

8 Winterthurerstrasse 190, CH-8057 Zurich, Switzerland

$9{ }^{4}$ Animal Ecology, Department of Ecology \& Genetics, Uppsala University, Uppsala, Sweden

$11 *$ Author for correspondence:

12 Research School of Biology, 116 Daley Road, Australian National University, ACT 0200

13 Australia

$14 \quad$ Ph.: +61 (2) 61256931

15 hanna.kokko@ieu.uzh.ch

16

17 SHORT TITLE: Costs and choosiness 


\section{Abstract}

19 Costly female mating preferences for purely Fisherian male traits (i.e. sexual ornaments that

20 are genetically uncorrelated with inherent viability) are not expected to persist at equilibrium.

21 The indirect benefit of producing 'sexy sons' (Fisher process) disappears: in some models the

22 male trait becomes fixed, in others a range of male trait values persist but a larger trait

23 confers no net fitness advantage because it lowers survival. Insufficient indirect selection to

24 counter the direct cost of producing fewer offspring means that preferences are lost. The only

25 well-cited exception assumes biased mutation on male traits. The above findings generally

26 assume constant direct selection against female preferences (i.e., fixed costs). We show that if

27 mate sampling costs are instead derived based on an explicit account of how females acquire

28 mates, an initially costly mating preference can coevolve with a male trait so that both persist

29 in the presence or absence of biased mutation. Our models predict that empirically detecting

30 selection at equilibrium will be difficult, even if selection was responsible for the location of

31 the current equilibrium. In general, it appears useful to integrate mate sampling theory with

32 models of genetic consequences of mating preferences: being explicit about the process by

33 which individuals select mates can alter equilibria. 


\section{Introduction}

36 The occurrence of extravagant sexual traits, usually in males, is an obvious feature of the

37 natural world. In some cases these traits are functional weapons (e.g. horns and tusks). In

38 other cases they are, however, purely ornamental (e.g. elongated tails, elaborate song, bright

39 plumage). It is widely accepted that ornaments have evolved under sexual selection driven by

40 female mate choice. One problem with this explanation is that extravagant male traits seem to

41 be most common in species where the rewards of choosiness for females appear to be

42 smallest. Specifically, in non-territorial species lacking male parental care, all that females

43 appear to gain from males is sperm. The advantage of being choosy therefore seems to be

44 associated with genetic (indirect) benefits that elevate net offspring fitness. This has led to a

45 theoretical challenge often referred to as the 'lek paradox' (Kirkpatrick \& Ryan, 1991).

46 It is challenging to explain costly female mating preferences for ornamented males when

47 females gain no direct benefits from choice. Fisher (1930) reasoned that choosy females

48 indirectly benefit by producing 'sexy sons' (i.e. males with above average mating success).

49 Indirect selection on the preference is based on a genetic correlation that arises between the

50 female preference and the preferred male trait: choosy females prefer ornamented males, and

51 there is direct positive selection on the male trait when it confers a sufficiently strong mating

52 advantage to outweigh reduced male survival. A breakthrough in sexual selection theory was

53 the validation of Fisher's process using quantitative and population genetic models of

54 preference-trait coevolution (Lande, 1981; Kirkpatrick, 1982).

55 An assumption of these early models was that female choice is cost-free. This is an important

56 assumption because once male trait expression reaches equilibrium, the indirect benefits of

57 choosiness disappear. There is no longer additive genetic variation in fitness associated with

58 the expression of the male trait. This occurs either because the trait goes to fixation, or, when 
59 variation in trait expression remains, there is a perfect trade-off between its beneficial effect

60 on male mating success and its detrimental effect on male survival (Lande, 1981). The net

61 result is that choosy females no longer produce fitter sons, which eliminates indirect selection

62 for the mating preference. At this point any costs of choosiness lead to direct selection against

63 female mating preferences, which decline to their naturally selected optimum (usually

64 assumed to be random mating). The male trait is consequently not maintained either.

65 The best-known solution allowing a costly mating preference to persist is to invoke biased

66 mutation on preferred male traits, thereby maintaining indirect benefits of choice

67 (Pomiankowski et al., 1991). Biased mutation maintains additive genetic variation in fitness

68 despite a directional mating preference. This demonstrates that Fisherian models can work

69 when choosing is costly, but these costs have to be minute given realistic mutation rates. It

70 seems that additional direct benefits and/or indirect viability benefits of choosiness are

71 required (Maynard Smith et al., 1991; Kuijper et al., 2012).

72 Frequency-dependent benefits are integral to female-choice models. Specifically, the

73 magnitude of the mating advantage of ornamented males depends on the relative frequency of

74 females with a mating preference that makes them more likely to mate with these males.

75 Strangely, however, despite early theoretical discussion of how preference expression might

76 depend on mate sampling tactics (O’Donald, 1980; Seger, 1985), equivalent frequency-

77 dependent costs of choosiness have attracted little attention. A notable exception is

78 Pomiankowski (1988) who showed that frequency-dependent changes in the costs of mating

79 preferences can alter equilibria. However, this early work made the verbal argument that

80 because random mating with the first male encountered is the least costly option, it cannot be

81 invaded by any other type of mate choice. Consequently, evolutionary competition between

82 two types of non-random mating (preference for either ornamented or for non-ornamented 
83 males) was modelled, such that preferences for the more common type of male were directly

84 selected for.

85 This study therefore deferred exploration of the crucial contrast between having a preference 86 and mating randomly, which is at the heart of most mate choice evolution models (i.e. why

87 preferences evolve when direct selection favours random mating). We show here that

88 frequency-dependent costs of choosiness merit reconsideration: even if direct selection never

89 favours preference alleles over random mating, the evolutionary dynamics do not always

90 predict preference erosion. Costs can exist everywhere along the coevolutionary path towards

91 the endpoint, which then, at equilibrium, features female choice that minimizes costs (as in

92 Pomiankowski 1988). Importantly, preferences can evolve upwards along this path despite

93 there being (continually diminishing) costs, because benefits have not yet vanished either.

94 Empiricists emphasize that the costs of choosiness depend on the effort expended in mate

95 searching and sampling: how long does it take, or how far must females travel, to find a

96 suitable mate? Theoretical models, however, typically use fixed costs that depend only on

97 whether a female carries a preference allele, or genes for greater expression of a preference

98 (for an exception in a good genes context see Houle \& Kondrashov (2002); in a speciation

99 context see Gavrilets \& Boake (1998) who include the cost of remaining unmated if the

100 preferred male type is rare). We investigate a simple scenario where it proves important to

101 take into account that the cost of a preference might change with the frequency of preferred

102 males. This should affect how the net benefits of choosiness change during the

103 coevolutionary process, with potential implications for evolutionary dynamics and for

104 evolutionary stable outcomes.

105 The fact that theoreticians have tended to ignore how relative encounter rates with preferred

106 and non-preferred males affect the costs of choosiness is a surprising omission, especially 
107 given the following statement by Andersson (1994, p. 43) in a book that almost every

108 researcher working on sexual selection has read:

109 "Preferences therefore may have a cost that is inversely related to the frequency of the

110 preferred type of male. The dynamics and stability conditions of the system then change

111 dramatically..."

112 Despite this statement, the lack of research interest in this area is reflected in the fact that

113 twelve of the thirteen well-known papers Andersson then cited (e.g. Lande, 1981;

114 Kirkpatrick, 1982; Pomiankowski et al., 1991; Seger, 1985) actually did not model

115 frequency-dependent costs (the sole exception being Pomiankowski, 1988). This is intriguing

116 because formal Fisherian models appeared simultaneously alongside a burst of research

117 investigating how mate sampling rules and preference functions affect mate quality (e.g.

118 Janetos, 1980; Parker, 1983; Real, 1990). Subsequent work has not unified these subfields.

119 Numerous theoretical studies have now considered the details of how mate sampling impacts

120 the expected 'quality' (or trait value) of chosen males (Sullivan, 1994; Luttbeg, 1996;

121 Mazalov et al., 1996; Wiegmann et al., 1996, 2010a,b, 2013; Johnstone, 1997; Hutchinson \&

122 Halupka, 2004; Wiegmann \& Angeloni, 2007), but these appear not to have influenced

123 theoretical work on the coevolution of preferences and traits. Even papers that involve mate

124 sampling costs (e.g. Houle \& Kondrashov, 2002) rarely cite this parallel literature as a source

125 of inspiration.

126 What would have happened if mate sampling theory had been formally incorporated into

127 early Fisherian mate choice models? Here we show that it might have discouraged the

128 textbook dogma that Fisherian benefits cannot, even in principle, sustain mating preferences

129 if they impose costs (e.g. Cameron et al., 2003). In the context of female choice for 'good

130 genes' (i.e. male viability indicator traits), a model that explicitly considered changes in mate 
131 sampling costs (Houle \& Kondrashov 2002) yielded different conclusions to those reached

132 with a model invoking fixed costs of mating preferences (Kirkpatrick 1996) (although note

133 that there are additional differences between these models).

134 Our aim here is to construct a parallel comparison between an early model of Fisherian

135 evolution (Kirkpatrick, 1982) and an approach that derives costs through explicitly modelled

136 mate sampling. We do this in two ways: (i) by a two-locus model with all assumptions

137 identical to early work, apart from introducing costs of choice that are based on explicit mate

138 sampling, and (ii) by building an individual-based model that relaxes many potentially

139 restrictive assumptions at once. We show that when choosy females pay smaller search costs

140 as preferred males become more common, the outcome of preference-trait coevolution

141 models changes dramatically. Contrary to early suggestions (Pomiankowski 1988), this can

142 happen even if choice is never cheaper than random mating.

\section{Methods}

Model 1: a two-locus model

145 Kirkpatrick (1982) provided a simple way to model indirect benefits given a female

146 preference $(P)$ for a male display trait $(T)$ that reduces male viability. We follow this general

147 approach. We assume a haploid population with discrete generations. Individuals of both

148 sexes have two loci; one determines the presence $\left(T_{1}\right)$ or absence $\left(T_{0}\right)$ of a male-only display

149 trait, the other determines whether a female preference for $T_{1}$ males is present $\left(P_{1}\right)$ or absent

$150\left(P_{0}\right)$. Females mate once. The display trait is costly: the viability of $T_{0}$ males is 1 , that of $T_{1}$

151 males is $1-s(s>0)$. Both indirect and direct selection act on female preferences. We

152 consider three versions:

153 (a) Full model: Following suggestions that realistic mate choice might only involve sampling 154 a few males (Roff \& Fairbairn, 2014), we assume $P_{1}$ females sequentially sample up to five 
155 males, while $P_{0}$ females mate with the first male encountered. If $q$ is the frequency of $T_{1}$

156 males after viability selection, the probability that a $P_{0}$ female mates with a $T_{1}$ male is $q$. For

157 a $P_{1}$ female, we assume that she mates as soon as she encounters a $T_{1}$ male, but if this has not

158 happened by her fifth mating encounter, she accepts the current $\left(T_{0}\right)$ male. Hence:

$$
\text { Prob }\left\{\text { sire is } T_{1} \mid \text { female is } P_{1}\right\}=q+(1-q) q+(1-q)^{2} q+(1-q)^{3} q+(1-q)^{4} q
$$

160 A $P_{0}$ female pays no cost of choosing as she always mates with the first male encountered.

161 For a $P_{1}$ female, the cost of choosing depends on how many males she samples before

162 mating. The full cost (denoted $c$ ) is paid only if she mates with the fifth male encountered,

163 while there is no cost if she mates at her first encounter (i.e., the same as for a $P_{0}$ female).

164 Assuming additively accumulating costs per sampling event, the expected cost for a $P_{1}$

165 female mating with a $T_{1}$ male is

166

$$
C=\frac{\frac{c}{4}(1-q) q+\frac{2 c}{4}(1-q)^{2} q+\frac{3 c}{4}(1-q)^{3} q+c(1-q)^{4} q}{\operatorname{Prob}\left\{\text { sire is } T_{1} \mid \text { female is } P_{1}\right\}}
$$

167 A $P_{1}$ female mating a $T_{0}$ male must have sampled five males, so her cost is always $c$.

168 (b) Hybrid model: sire identity is determined following the full model. $P_{0}$ females again pay

169 no sampling cost, but $P_{1}$ females always pay the full cost $c$. Because the hybrid model

170 combines an explicit sampling process with the assumption of classic models of female

171 choice that costs of choosiness are unavoidable, comparing the outcomes of the hybrid model

172 with the full model and the classic model (see below) helps to disentangle the independent

173 roles of cost frequency-dependence and the effects of sampling on mate choice evolution.

174 (c) Classic model: a $P_{1}$ female mates with a $T_{1}$ male with probability

175

$$
\operatorname{Prob}\left\{\text { sire is } T_{1} \mid \text { female is } P_{1}\right\}=\frac{a q}{a q+(1-a)},
$$


176 where $a$ is equivalent to $a_{2}$ in Kirkpatrick (1982) (one interpretation is that a $P_{1}$ female is $a$

177 times more likely to mate a $T_{1}$ than $T_{0}$ male in a two-choice test). The probability that a $P_{0}$

178 female mates with a $T_{1}$ male is $q$. All $P_{1}$ females pay the full $\operatorname{cost} c$.

179 The models track genotype frequencies in each generation (see Appendix), assuming the 180 order is: (1) viability selection on $T_{1}$ males; (2) females choose mates; (3) females breed

181 (given a cost $C$ or $c$, female fecundity is multiplied by $(1-C)$ or $(1-c)$ ); (4) parents die. We

182 also included an option at step (4) for biased mutation converting $T_{1}$ to $T_{0}$ (i.e. trait loss) at

183 the rate $\mu$. This allows us to contrast our results with models where biased mutation maintains

184 female preferences (Pomiankowski et al., 1991).

\section{Model 2: an individual-based simulation}

Kuijper et al. (2012) review four different approaches to implementing the Fisher process. To check whether the gist of our argument based on the simplest approach holds, we use the last, and most complex, approach: an individual-based simulation (see also Roff \& Fairbain, 2014). The most important assumption to relax is the dichotomous nature of ornaments and preferences. In the individual-based simulation, each individual is characterized by its sex, by 100 haploid loci with alleles 0 or 1 for an additive male trait $T$ (expressed only in males), and one haploid allele that takes positive integer values and specifies the female preference threshold $P$ (expressed only in females). We assume a mutation rate $\mu$ for the female preference and $\mu / 100$ for each locus of the male trait (thus leading to the same overall mutation pressure for preferences and traits alike). Female choice requires that males exceed a threshold trait value: a female with preference $P$ will mate with males whose sum of allelic values is at least $P$. Mate sampling occurs as in the full and hybrid two-locus models, with costs accumulating in the same way such that females who reach the maximum number of mates sampled $(N)$ pay the highest cost. As a further check of robustness, we combine results 
200 derived with $N=5$ (as in the two-locus models) with others that use $N=10$. Details of the

201 model are given in the Electronic Appendix.

\section{Results and Discussion}

203 As in all Fisherian models, the mating preference must initially exceed a threshold frequency

204 (invasion barrier) in order to increase and be maintained by indirect selection (Fig. 1: green

205 area). We considered six scenarios in the two-locus model (the full, hybrid, and classic model

206 variants, either with or without biased mutation on the male trait). For easier visualization in

207 Fig. 1, we use relatively large values for both the maximum cost of the female preference $(c=$

208 0.01: sampling five males reduces female fecundity by $1 \%)$, and the cost of the male trait $(s=$

209 0.4: $T_{1}$ males have 40\% lower survival). Smaller costs lower the invasion barrier to

210 preference (and trait) increase and maintenance. For example, if $c=0.001$ and $s=0.1$, an

211 initial preference prevalence of $4 \%$ is sufficient to lead to trait maintenance (if the male trait

212 has $\geq 2 \%$ prevalence initially).

213 We first consider what happens without biased mutation on the male trait. When sampling

214 costs decline as the frequency of preferred $T_{1}$ males increases, the male trait goes to fixation if

215 the initial frequency of the preference exceeds the invasion barrier (the full model, Fig. 1a).

216 At equilibrium there is no direct selection on the mating preference: all males are of the

217 preferred type, so both $P_{0}$ and $P_{1}$ females mate with the first male they encounter. As $T_{1}$ is

218 fixed, there is also no longer indirect selection on the preference allele. This results in a

219 neutral line of equilibria (see Appendix). In contrast, when sampling costs are independent of

220 the frequency of $T_{1}$ males, a costly preference, and hence costly male display, cannot persist

221 at equilibrium (both the classic and hybrid models, Fig. 1c). The crucial difference is

222 therefore due to the frequency-dependence of the costs of choice, and not to how the mate- 
223 sampling tactic determines mate identity: although the hybrid model uses an explicit

224 sampling process, the fixed cost of choosiness ensures neither the preference nor trait persist.

225 A purely Fisherian process (indirect selection on a mating preference arising solely from

226 linkage disequilibrium with a preferred male trait under direct selection) can thus maintain a

227 costly male sexual display even when the preference carries costs due to active mate

228 sampling, and when random mating is included as a 'cheap' alternative. Previously the

229 evolutionary stability of a preference for such Fisherian traits has only been demonstrated

230 when there is biased mutation on the male trait (e.g. Pomiankowski et al., 1991).

231 In the individual-based model, where we relax the assumption that male traits and female

232 preferences are dichotomous variables, the preference thresholds used by females evolve to

233 be consistently below the mean of the male trait distribution (see the Electronic Appendix).

234 Preferences and traits coevolve until they drift along a line that appears - to the extent that it

235 is possible to deduce this from an individual-based simulation - neutral. In the depicted

236 trajectories of Fig. 2, we found no evidence of preference decay, and neither were collapsing

237 cases found when we tried starting simulations from 50 randomly chosen parameter settings

238 (Fig. 2, shaded area). Although the dynamic consequences of a preference threshold clearly

239 differ from those arising from the simpler preferences implemented in the full two-locus

240 model, the findings of both models are consistent with our general interpretation: preferences

241 can evolve upwards when both benefits and costs are significant, and this directional

242 evolution stops once males have evolved to 'satisfy' female preferences and costs have

243 become irrelevant.

244 We are aware that it is tempting to dismiss our findings as somehow trivial because the

245 mating preference becomes cost-free at equilibrium. We argue that this would be uncharitable

246 as: (i) the preference imposes costs at every point on the evolutionary trajectory towards 
247 equilibrium, so the situation modelled is not analogous to that of classic cost-free models

248 (e.g. Kirkpatrick, 1982); (ii) the biological reality is that preference costs are rarely

249 independent of the frequency of preferred males, so it is an artificial construct to maintain a

250 cost where none would exist; (iii) it is not obvious a priori that the rate at which direct

251 selection against a preference declines can be greater than the rate at which indirect benefits

252 decline (due to less variation in attractiveness as $T_{1}$ approaches fixation); (iv) intriguingly the

253 model accounts for male traits persisting despite no current advantage to choosiness.

254 We next consider what happens given biased mutation on the male trait. In the examples

255 presented (Fig. 1b, d) the mutation rate is low $(\mu=0.0001)$. When sampling costs decline as

256 preferred $T_{1}$ males increase in frequency, becoming easier to find, there are only two

257 equilibrium points (the full model, Fig. 1b). Again, if the initial preference frequency lies

258 below the invasion barrier, the preference and trait are eliminated. If above the invasion

259 barrier, however, the preference becomes fixed while the male trait almost reaches fixation

260 (the proximity depends on $\mu$ : the lower it is, the closer the trait is to fixation). The indirect

261 benefit of choosiness (due to variation in male genotypes, $q<1$ ) exceeds, in this example, the

262 strength of direct selection, so the preference increases in frequency until it reaches fixation.

263 Again, it was unclear a priori that the relative rate of decline in direct selection could exceed

264 that of indirect selection.

265 It is well established theoretically that biased mutation can sustain a costly preference

266 (Pomiankowski et al., 1991). In our example, a frequency-independent cost of choice

267 eliminates both the preference and male trait (the hybrid and classic models, Fig. 1d). Of

268 course, we emphasize that this would not have occurred had we used a sufficiently high

269 mutation rate. The point we wish to make, however, is that a mutation rate too low to 
270 maintain a preference with a fixed cost (Fig. 1d) can maintain it when costs are explicitly

271 derived from mate sampling theory (Fig. 1b).

272 Our model does not explain how a mating preference initially increases in frequency to 273 exceed an invasion barrier. Nor does it fully resolve the lek paradox (why females are choosy

274 if choice depletes variation), as it ignores non-additive and environmental factors that affect male trait expression. This is a potential limitation of the model because such factors reduce indirect selection on the mating preference (i.e. choosy females less often acquire fitter sons), but it should be noted that most models of preference-trait coevolution similarly avoid this 278 complication.

279 For empiricists it is worth reflecting on the difference in observations when viewing a population moving towards equilibrium (A) and another already at equilibrium (B). In $\mathrm{A}$ it is possible to detect costs of choosiness that are up to $1 \%$ of a female's fecundity, and observe that the mating success of sons depends on their father's genotype $\left(T_{0}\right.$ or $\left.T_{1}\right)$. In contrast, in B all males have a costly trait, and most (Fig. 1a) or all (Fig. 1b) females have a cost-free mating preference for this trait (demonstrable by experimental manipulation of trait expression to induce the requisite variation). Despite this, there is no measurable advantage for the sons of females that mated with preferred males. It would obviously be puzzling to field researchers as to why females have evolved to prefer ornamented males in population B.

Our model is a reminder that variation among males in the evolutionary past could have created coevolutionary forces that drove female preferences to their current levels, where they are now hard to explain. Preferences become non-neutral again, re-establishing indirect

291 selection and observable trait-preference coevolution, as soon as populations are pushed to

292 the interior of Fig. 1. This would occur, for example, if increased mutation rates reduce the 
293 male trait. It is possible that empirical observations of female choice for currently non-

294 beneficial male traits partially reflect 'the ghost of selection past'.

295 In sum, much of the frustration in testing sexual selection theory hinges on the prediction that 296 direct selection against mating preferences easily outweighs indirect selection to mate non-

297 randomly. Our results reinforce this message: minute differences in direct costs affect our

298 ability to explain male traits (e.g. the classic two-locus model; Fig. 1), but our models also

299 include cases where predictable variation in costs becomes essential for the maintenance of a

300 preference.

301 The as such correct statement, that it is difficult to explain costly female choice for indirect

302 benefits, should therefore be accompanied by reminders that the magnitude of benefits and

303 costs can change dynamically during preference-trait coevolution, and that these changes do

304 not necessarily occur at the same rate. If costs diminish faster than benefits, the evolutionary

305 outcome can be far more choosiness than if the opposite is true. For empiricists, there is

306 obvious potential to quantify the magnitude of frequency-dependent changes in the costs of

307 choosiness by experimental manipulation of the frequency of different male types. One of the

308 key messages, however, is that this might be a difficult task: we predict preferences will

309 persist precisely where evolution has led the currently expressed costs to be minimal.

\section{Acknowledgements}

311 We thank Andrew Pomiankowski and an anonymous reviewer for their insightful comments,

312 and the Australian Research Council and the Academy of Finland for funding.

\section{References}

314 Andersson, M. 1994. Sexual Selection. Princeton University Press, Princeton. 
315 Cameron, E., Day, T. \& Rowe, L. 2003. Sexual conflict and indirect benefits. J. Evol. Biol.

316 16: 1055-1060.

317 Fisher, R.A. 1930. The Genetical Theory of Natural Selection. Clarendon, Oxford.

318 Gavrilets, S. \& Boake, C.R.B. 1998. On the evolution of premating isolation after a founder

319 event. Am. Nat. 152: 706-716.

320 Houle, D. \& Kondrashov, A.S. 2002. Coevolution of costly mate choice and condition-

321 dependent display of good genes. Proc. R. Soc. Lond. B 269: 97-104.

322 Hutchinson, J.M.C. \& Halupka, K. 2004. Mate choice when males are in patches: optimal 323 strategies and good rules of thumb. J. Theor. Biol. 231:129-151.

324 Janetos, A.C. 1980. Strategies of female mate choice: a theoretical analysis. Behav. Ecol.

325 Sociobiol. 7: 107-112.

326 Johnstone, R.A. 1997. The tactics of mutual mate choice and competitive search. Behav.

327 Ecol. Sociobiol. 40:51-59.

328 Kirkpatrick, M. 1982. Sexual selection and the evolution of female choice. Evolution 36: 1-

32912.

330 Kirkpatrick, M. 1996. Good genes and direct selection in the evolution of mating preferences.

331 Evolution 50: 2125-2140.

332 Kirkpatrick, M. \& Ryan, M.J. 1991. The evolution of mating preferences and the paradox of 333 the lek. Nature 350: 33-38.

334 Kokko, H., Jennions, M.D. \& Brooks, R. 2006. Unifying and testing models of sexual 335 selection. Annu. Rev. Ecol. Evol. Syst. 37: 43-66. 
336 Kuijper, B., Pen, I. \& Weissing, F.J. 2012. A guide to sexual selection theory. Annu. Rev.

337 Ecol. Evol. Syst. 43: 287-311.

338 Lande, R. 1981. Models of speciation by sexual selection on polygenic traits. Proc. Natl.

339 Acad. Sci. USA 78: 3721-3725.

340 Luttbeg, B. 1996. A Comparative Bayes tactic for mate assessment and choice. Behav. Ecol.

$341 \quad 7: 451-460$.

342 Mazalov, V., Perrin, N. \& Dombrovsky, Y. 1996. Adaptive search and information updating

343 in sequential mate choice. Am. Nat. 148:123-137.

344 Maynard Smith, J. 1991. Theories of sexual selection. Trends Ecol. Evol. 6: 146-151.

345 O'Donald, P. 1980. Genetic Models of Sexual Selection. Cambridge University Press,

346 Cambridge.

347 Parker, G.A. 1983. Mate quality and mating decisions. In: Mate Choice (P. Bateson, ed.), pp.

348 141-166. Cambridge University Press, Cambridge.

349 Pomiankowski, A. 1988. The evolution of female mate preferences for male genetic quality.

350 Oxf. Surv. Evol. Biol. 5: 136-184.

351 Pomiankowski, A., Iwasa, Y. \& Nee, S. 1991. The evolution of costly mate preferences I.

352 Fisher and biased mutation. Evolution 45: 1422-1430.

353 Real, L. 1990. Search theory and mate choice. I. Models of single-sex discrimination. Am.

354 Nat. 136: 376-405.

355 Rice, S.H. 2004. Evolutionary Theory. Mathematical and conceptual foundations. Sinauer, 356 Sunderland. 
357 Roff, D.A. \& Fairbairn, D.J. 2014. The evolution of phenotypes and genetic parameters under 358 preferential mating. Ecology and Evolution (in press).

359 Seger, J. 1985. Genetic models for the evolution of female choice. Evolution 39: 1185-1193.

360 Sullivan, M.S. 1994. Mate choice as an information gathering process under time constraint -

361 implications for behavior and signal-design. Anim. Behav. 47:141-151.

362 Wiegmann, D.D. \& Angeloni, L.M. 2007. Mate choice and uncertainty in the decision 363 process. J. Theor. Biol. 249:654-666.

364 Wiegmann, D.D., Angeloni, L.M. \& Seubert, S.M. 2013. Mate choice decisions by searchers.

365 Curr. Zool. 59:184-199.

366 Wiegmann, D.D., Real, L.A., Capone, T.A. \& Ellner, S. 1996. Some distinguishing features

367 of models of search behavior and mate choice. Am. Nat. 147:188-204.

368 Wiegmann, D.D., Seubert, S.M. \& Wade, G.A. 2010a. Mate choice and optimal search

369 behavior: Fitness returns under the fixed sample and sequential search strategies. $J$. Theor.

370 Biol. 262: 596-600.

371 Wiegmann, D.D., Weinersmith, K.L. \& Seubert, S.M. 2010b. Multi-attribute mate choice

372 decisions and uncertainty in the decision process: a generalized sequential search strategy. $J$.

373 math. Biol. 60: 543-572. 


\section{Appendix}

376 Our two-locus model largely follows the derivation of Kirkpatrick (1982), which is

377 beautifully explained in expanded form (including mutations) in chapter 2 of Rice (2004).

378 Populations in Fig. 1 are initialized assuming no linkage disequilibrium. Thus, if the initial

379 frequency of the preference is assumed to be $x$ and that of the male trait to be $y$, genotype

380 frequencies in the initial generation are

$$
p_{00}=(1-x)(1-y), p_{01}=(1-x) y, p_{10}=x(1-y), p_{11}=x y
$$

where the first subscript denotes the value of the $P$ allele, and the second refers to the $T$ allele.

383 Each generation then first applies viability selection, which modifies the frequency of $T_{1}$

384 males in the current generation to

$$
q=\frac{(1-s)\left(p_{11}+p_{01}\right)}{(1-s)\left(p_{11}+p_{01}\right)+\left(p_{00}+p_{10}\right)}
$$

This value of $q$ is then used to calculate mate identities and costs paid by females (equations $1-3$ in main text).

Thereafter, offspring production follows the probabilities of each female finding a sire of each genotype, and Mendelian laws of genetics. Consider, for example, the production of $P_{1} T_{1}$ offspring (the bookkeeping for the three other offspring types proceeds similarly). All matings between $P_{1} T_{1}$ females and $P_{1} T_{1}$ males lead to $P_{1} T_{1}$ offspring (before mutation). But so do half of the offspring produced in matings between $P_{1} T_{1}$ females and $P_{1} T_{0}$ males, between $P_{1} T_{0}$ females and $P_{1} T_{1}$ males, between $P_{0} T_{1}$ females and $P_{1} T_{1}$ males, or between $P_{1} T_{1}$ females and $P_{0} T_{1}$ males. Finally, one quarter of the offspring produced by $P_{1} T_{0}$ females mating with $P_{0} T_{1}$ males, and of matings between $P_{0} T_{1}$ females mating with $P_{1} T_{0}$ males, also

396 become $P_{1} T_{1}$. The proportions are multiplied by $1-c$ or $1-C$ where relevant: for example, 397 neither cost applies to $P_{0} T_{1}$ females mating with any kind of male. 
398 Thereafter, mutations occur: a proportion $\mu$ of $P_{1} T_{1}\left(P_{0} T_{1}\right)$ offspring become $P_{1} T_{1}\left(P_{0} T_{0}\right)$.

399 After normalizing, the new generation is ready.

400 The model yields, in principle, an analytic expression for the change of allele frequencies, but 401 in the interior of Fig. 1 the expressions are too unwieldy to yield much insight, and are not 402 reproduced here (the Mathematica file is available upon request). However, they simplify to 403 zero when assumption set (a) is combined with $p_{01}=p_{11}=0$, which indicates that the female 404 preference becomes neutral when the male trait is fixed. This makes intuitive sense, as in that 405 case the realized mating behaviour does not differ between choosy and randomly mating 406 females (neither in terms of mate identity nor the sampling effort taken). 
408 Fig. 1. Frequencies of preference allele $\left(P_{1}\right)$ and $T_{1}$ males after viability selection in the two-

409 locus models. Evolutionary trajectories (arrowed lines) always start from the same four

410 representative points. Solid circles indicate evolutionary endpoints (equilibria). The full

411 model output is in (a) and (b), showing that the system can equilibrate with positive traits and

412 preferences. The green area indicates initial combinations that generate a coevolutionary

413 increase in $P_{1}$ and $T_{1}$ towards such equilibria. Such combinations are completely absent in the

414 hybrid model (solid lines) and classic model (dotted line), depicted in (c) and (d): thus

415 constant costs rather than the effect of sampling on mate identity is responsible for the

416 difference in outcome. Biased mutation on male traits is either absent $(\mathrm{a}, \mathrm{c})$ or present at $\mu=$

$4170.0001(b, d)$.

418 Fig. 2. Coevolution of female preference thresholds and male traits in the individual-based

419 simulation, with $c=0.01, \mu=0.01$ and either a maximum of $N=5$ sampled males ( 11

420 depicted trajectories) or $N=10$ (the 50 trajectories are not depicted, location of all endpoints

421 indicated by the green shaded area). We depict 11 trajectories that start at the black squares as

422 indicated (to cover a range of values where either the preference or the trait is not yet strong),

423 and end at the red stars at generation 10000. For clarity in a simulation that was run for 10000

424 generations per trajectory, the lines track the mean allelic values of each population only

425 every 100 generations. To increase the robustness of the conclusion that simulations do not

426 collapse, and that preferences and traits can coevolve to higher values their initial starting

427 values, we ran our $N=10$ (maximum number of males sampled) examples from random

428 starting points: each case was started such that mean female preference threshold was

429 randomized to be between 0 and 50, and mean male traits were between 0 and 90 . None of

430 the outcomes at generation 10000 were found outside the green shaded area. The overall

431 conclusion is that all populations evolve to spend much of the evolutionary time near a line

432 where the mean male trait values somewhat exceed the mean threshold mating preference 
433 values; some populations evolve males with strongly compromised survival (male traits

434 higher than 75 lead to more than $50 \%$ male viability reduction, and 8 out of 50 random runs

435 exhibited such values at generation $10000 ; 2$ of these had the trait persist at its maximum

436 where all 100 male trait alleles had the value 1). 


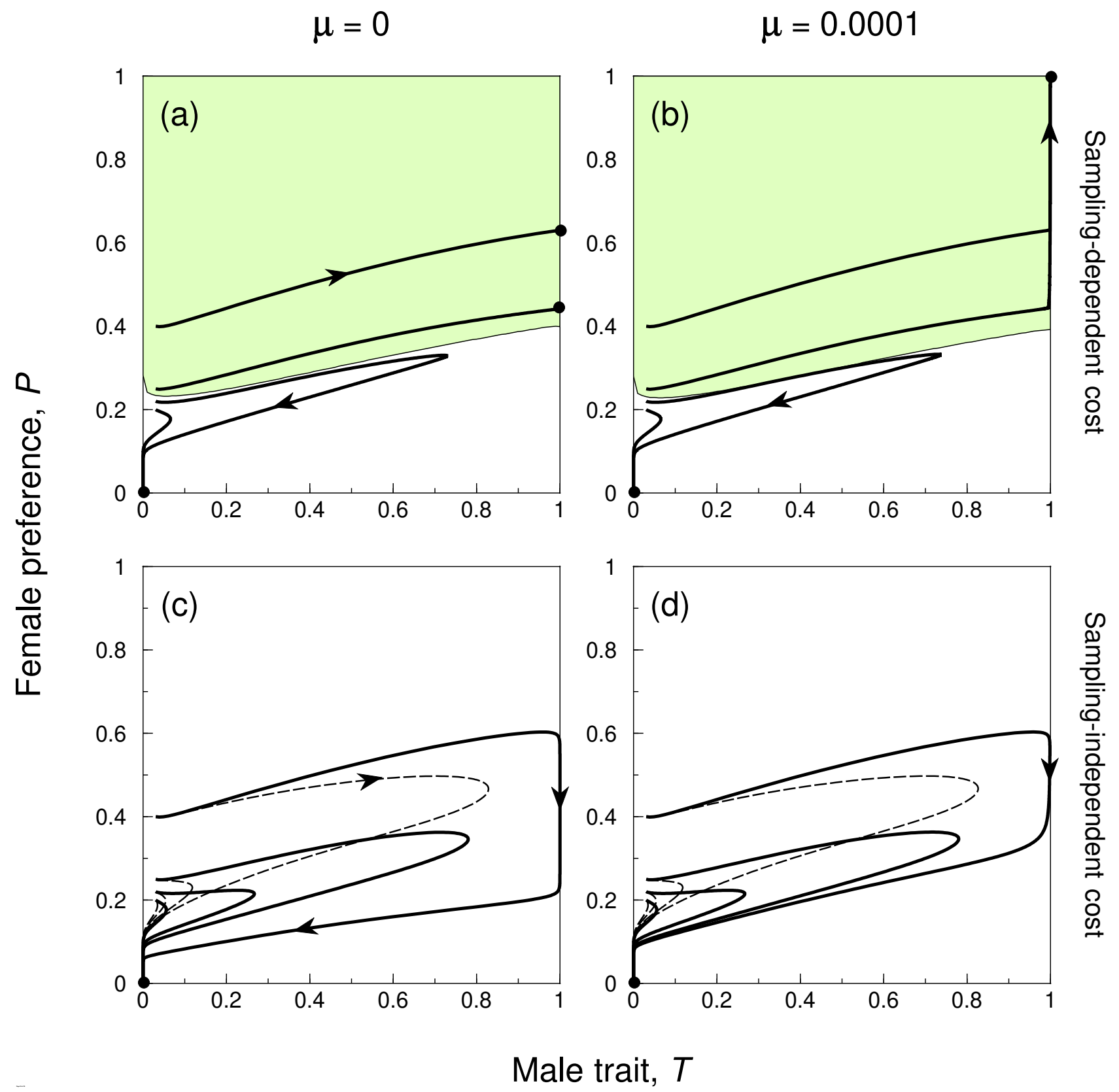




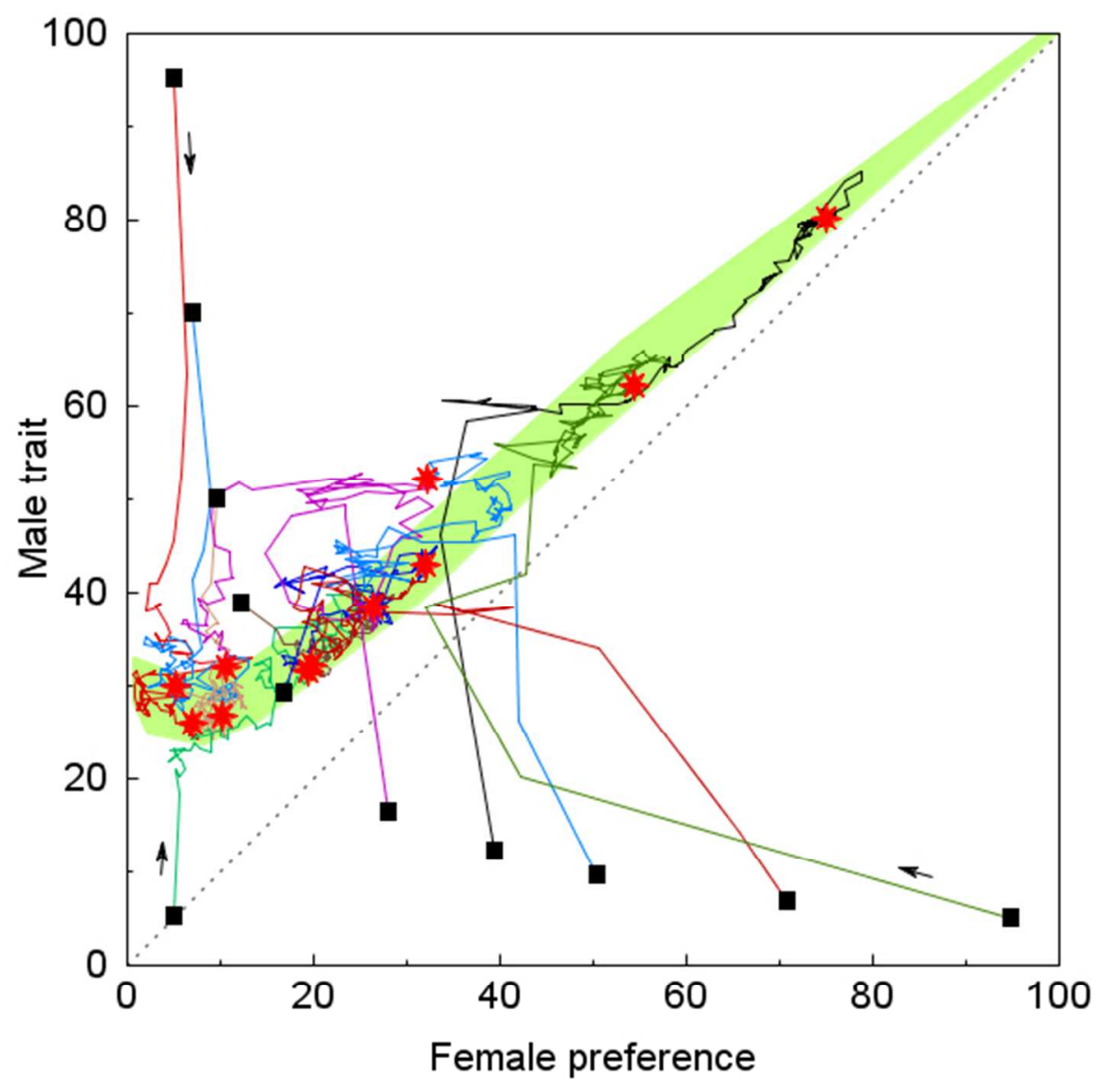

$227 \times 214 \mathrm{~mm}(72 \times 72 \mathrm{DPI})$ 


\section{Electronic appendix}

Our individual-based simulation is run in a population of 2000 individuals as follows:

1. Each individual is characterized by its sex (initially randomly determined), by 100 haploid loci with alleles 0 or 1 for the male trait $T$ (expressed only in males), and one haploid allele that takes integer values and specifies the female preference threshold $P$ (expressed only in females). Initially a proportion $0 \leq T_{0}<1$ of male trait alleles are set to 1 . Female alleles are set to uniformly distributed random values between 0 and $2 P_{0}$, implying that the mean preference threshold of the first generation will be $P_{0}$.

2. A generation is considered to begin at the start of the mating season. Each female is given a set of $N$ randomly chosen males to evaluate. She samples them one at a time until an acceptable one, i.e. one who has at least as many alleles of value 1 as the female's threshold implies, is found. Mate sampling stops at mating. If none of the males exceed the female threshold, she mates with the last male and is recorded to have sampled $N$ males.

3. A total of 2000 offspring are created. Each of them is assigned a mother and a father in the following way. Females compete for being a mother of a young, with competitiveness of each female being $1-c \frac{n-1}{N-1}$ if she sampled $n$ males out of the $N$ before mating. This implies that a female who mated with the first male $(n=1)$ pays no cost (her competitiveness is 1$)$, and a female who samples all $N$ males has her competitiveness reduced to $1-c$. Offspring are assigned to mothers probabilistically in proportion to mothers' competitiveness, which implies that a mother's fecundity is proportional to the competitiveness value she was potentially compromising through mate sampling. 
4. Offspring are created through Mendelian inheritance based on the mother's and the sire's known identities. Offspring sex is randomly determined. Parents die.

5. Preference alleles mutate. We assume a mutation rate $\mu$ for the female preference and $\mu / 100$ for each locus of the male trait (thus leading to the same overall mutation pressure for preferences and traits alike). Male trait mutations switch allelic values between 0 and 1 . Female preference mutations reduce or increase (50\% either way) the threshold by one, with the exception of mutations that would make the preference negative, which we assume have no effect.

6. Male traits reduce male survival. Each male survives with probability $1-\frac{1}{1+e^{-(T-75) / 10}}$ where $T$ is the sum of all his trait alleles. This function takes a declining logistic shape: small traits do not significantly harm survival, while 75 positive alleles are sufficient to halve his survival; however we do not assume that survival is completely impossible at any level of trait development:

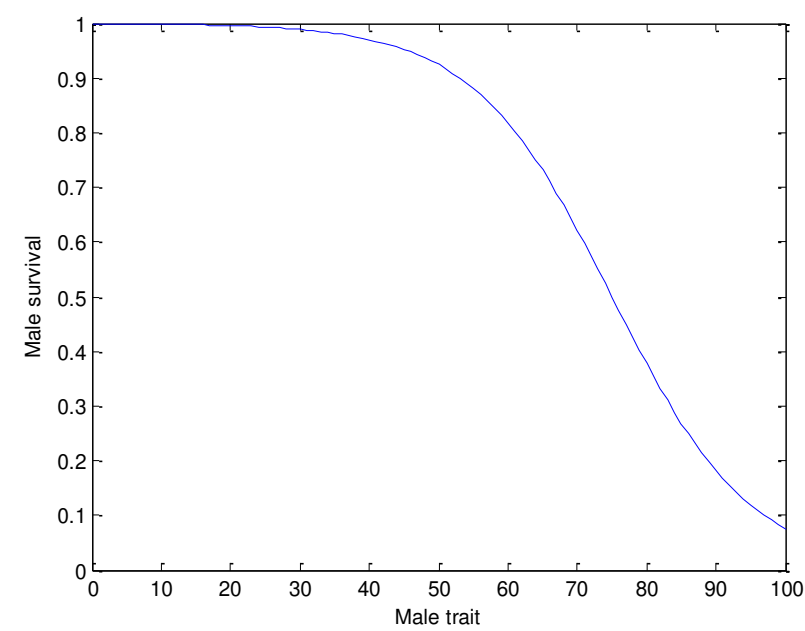

7. The new generation is ready to mate; we go to step 2 and repeat steps 2-7 for 10000 generations. 